\title{
Qualidade de vida no trabalho do enfermeiro atuante no bloco cirúrgico
}

\author{
Surgical ward nurse's quality of life at work of working
}

Calidade de vida trabalho del enfermeros que trabaja nuna unidad quirúrgica

Mayra Salgado de Lucena ${ }^{1 *}$, Helena Maria da Silva Oliveira ${ }^{2}$, Tereza Natália Bezerra de Lima ${ }^{3}$, Joelma Laurentino Martins de Souza ${ }^{3}$, Maria Eduarda de Lima Carvalho', llka Jenifer Menezes Taurino $^{4}$, Débora Rafaela Amorim Ferreira ${ }^{5}$, Deiviane Lucio Fernandes ${ }^{6}$, Andréia Patrícia Terenci ${ }^{7}$, lanne Larisse Alves Ferreira ${ }^{8}$, Fernanda Barbosa dos Santos ${ }^{9}$, Eliane Pereira do Nascimento ${ }^{10}$, Josevaldo Francisco da Silva ${ }^{11}$, Juliana de Oliveira Pimentel ${ }^{12}$, Júlia de Lima Cabral ${ }^{12}$.

\section{RESUMO}

Objetivo: Analisar a qualidade de vida no trabalho do enfermeiro atuante em um bloco cirúrgico. Método: Pesquisa quantitativa e descritiva, mediante uma entrevista estruturada com questionário de avaliação da Qualidade de Vida no Trabalho (QWLQ-78) validado e simplificado, para coletar dados dos enfermeiros, atuantes há mais de 6 meses no Bloco Cirúrgico. Resultados: Participaram da pesquisa 15 profissionais enfermeiros, $13(87 \%)$ pertencendo ao sexo feminino, $8(53 \%)$ trabalhadores de enfermagem com faixa etária entre 31 à 40 anos, nenhum filho, relacionado aos vínculos 11 (73\%) destes revelaram possuir 2 vínculos empregatícios e renda entre até 3 salários e acima de 5 salários mínimos. E a qualidade de vidas destes profissionais foi avaliada como mais ou menos de acordo com a escala de likert, por $9(60 \%)$ integrantes da pesquisa. Conclusão: Considerando que Qualidade de Vida no Trabalho (QVT) é um conjunto de elementos relacionados entre si e com isto obtivemos valores moderado como insatisfações para com a instituição e vida pessoal. Os resultados mostram que as percepções destes enfermeiros em sua maioria relataram de acordo com alguns pontos da Escala de Likert.

Palavras-chave: Enfermagem, Qualidade de vida, Centro cirúrgico, Bloco cirúrgico.

\footnotetext{
ABSTRACT

Objective: To analyze Surgical Ward Nurse's Quality of Life at work of working. Methods: Quantitative and descriptive research, through a structured interview with validated and simplified Quality of Life at Work assessment questionnaire (QWLQ-78) an interview with nurses, working for over 6 months in the Surgical

${ }^{1}$ Centro Universitário do Vale do Ipojuca (UNIFAVIP/Wyden), Caruaru-PE.

*E-mail: mayra lucena@hotmail.com

2Faculdade São Miguel (UNISÃOMIGUEL), Recife-PE.

3 Universidade de Pernambuco (UPE), Recife-PE.

${ }^{4}$ Centro de Formação e Aperfeiçoamento Profissional (CEFAPP), Recife-PE.

${ }^{5}$ Faculdades Integradas da Vitória de Santo Antão (FAINTVISA), Vitória de Santo Antão-PE.

${ }^{6}$ Centro Universitário São Camilo, Crato-CE.

${ }^{7}$ Centro de Formação e Aperfeiçoamento Profissional (CEFAPP), Recife-PE.

${ }^{8}$ Faculdade Metropolitana de Ciência e Tecnologia (FAMEC), Parnamirim-RN.

${ }^{9}$ Fundação de Ensino Superior de Olinda (FUNESO), Olinda -PE.

${ }^{10}$ Escola de Saúde Pública de Pernambuco (ESPPE/SES -PE), Recife - PE.

${ }^{11}$ UniversidadeTiradentes (UNIT), Recife - PE.

${ }^{12}$ Universidade Federal de Pernambuco (UFPE), Recife - PE.
} 
Block. Results: Participated in the research 15 professional nurses, $13(87 \%)$ female, $8(53 \%)$ nursing workers aged 31 to 40 years, no child, related to the links 11 (73\%) of these revealed to have 2 employment and income up to 3 wages and above 5 minimum wages. And the quality of life of these professionals was assessed as more or less by $9(60 \%)$ members of the research. Conclusion: Considering that Quality of Life at Work (QWL) is a set of elements related to each other and with this we obtained moderate values as dissatisfaction with the institution and personal life. The results show that the perception of these nurses mostly reported as more or less according to the likert scale.

Keywords: Nursing, Quality of life, Surgery center, Surgical ward.

\section{RESUMEN}

Objetivo: Analizar la calidad de vida en el trabajo de los enfermeros que trabajan en una sala de cirugía. Método: Investigación cuantitativa y descriptiva, a través de una entrevista estructurada con un cuestionario de evaluación de calidad de vida en el trabajo, validado y simplificado (QWLQ-78) con enfermeros, que trabaja durante más de 6 meses en el Bloque Quirúrgico. Resultados: Participaron en la investigación 15 enfermeros profesionales, $13(87 \%)$ mujeres, $8(53 \%)$ trabajadores de enfermería de 31 a 40 años, sin hijos, relacionados con los enlaces $11(73 \%)$ de estos revelaron tener 2 enlaces empleo e ingresos hasta 3 salarios mínimos y más de 5 salarios mínimos. Y la calidad de vida de estos profesionales fue evaluada como más o menos de acuerdo con la escala Likert por $9(60 \%)$ miembros de la investigación. Conclusión: Considerando que la Calidad de Vida en el Trabajo (QWL) es un conjunto de elementos relacionados entre sí y con esto obtuvimos valores moderados como insatisfacción con la institución y la vida personal. Los resultados muestran que las percepciones de estas enfermeras se informaron principalmente según algunos puntos de la escala Likert.

Palabras clave: Enfermería, Calidad de vida, Centro cirúrgico, Bloque quirúrgico.

\section{INTRODUÇÃO}

$\mathrm{Na}$ década de 1950, na Inglaterra, foi analisado um modelo para agregar o trinômio: Indivíduo/Trabalho/Organização. Essa inovação foi denominada de Qualidade de Vida no Trabalho (QVT). Contudo, o conceito de QVT mostra que é essencial humanizar o ambiente de trabalho, ofertando saúde e bem estar aos colaboradores (REZENDE KJ e GUARDA RM, 2017). O resultado dessa construção está diretamente ligado a esses fatores como satisfação, gerenciamento do trabalho e motivação (ADRIANO LGO, 2017). A satisfação nas atividades exercidas é gerada a partir de vários fatores que se interligam. Estes fatores relatam a respeito das normas organizacionais, status profissional, remuneração, requisitos do trabalho, interação e autonomia (SARTORETO IS e KURCGANT P, 2017).

A profissão de enfermagem é observada com poucos estudos sobre esta temática e submetida a inúmeros fatores que influenciam na qualidade de vida desses profissionais. Os fatores que interferem podem ser de fonte intrínseco ou extrínseco o que pode impactar na vida pessoal do profissional, dessa forma gerando como resposta a motivação (ADRIANO LGO, 2017).

Contudo, há relatos que ainda se encontra condições de trabalho desapropriadas à saúde destes colaboradores, acarretando a adoecer mentalmente e fisicamente. É necessário que o enfermeiro identifique como prioridade as condições oferecidas a eles, julgando primordialmente o fator das condições de trabalho. Assim, dar importância a este fator, no sentido de preservar a qualidade de vida e dedicar-se ao trabalho (ABBASI M, 2017).

Portanto possuir qualidade de vida no trabalho significa obter satisfação e motivação, nas atividades prestadas diariamente no emprego de modo que não associe como peso da desmotivação. Desse modo, as vivências do enfermeiro no Centro Cirúrgico ao desempenhar suas atribuições percebem a influência desse ambiente restrito, intenso e rotineiro, por isso sua competência e responsabilidade atrelada a exposição de agentes estressores decursivo dos vínculos interpessoais, ausência equipamentos para o trabalho, déficit de 
profissionais qualificados, demanda alta de cirurgias, estado fisiológico e mental dos pacientes. Assim, profissionais expostos a cada plantão a essas condições, estas poderão repercutir em seu estado de bem estar como também,em sua qualidade de vida no trabalho.Pois como evidenciado, destaca-se a relevância do trabalhador encontrar satisfação, com intenção de realizar melhor a responsabilidade profissional e ampliar sua percepção sobre qualidade de vida no trabalho (FU CY,et al., 2018).

Por isso, o objetivo do presente estudo foi analisar a qualidade de vida no trabalho do enfermeiro atuante em um bloco cirúrgico, compreender como se encontra a qualidade de vida no trabalho do enfermeiro atuante em um bloco cirúrgico bem como descrever o perfil sócio demográfico da população estudada. Além disso, buscou-se avaliar a percepção do enfermeiro relacionada à satisfação na qualidade de vida e no trabalho e identificar o nível de preocupação do enfermeiro do Bloco Cirúrgico em relação à auto avaliação na qualidade de vida no trabalho de acordo com a Escala de Likert.

\section{MÉTODOS}

Tratou-se de um- estudo com abordagem quantitativa, descritivo e transversal, em um hospital regido pelo Sistema Único de Saúde (SUS) localizado a uma cidade em Pernambuco. A coleta de dados aconteceu no período de Abril e Maio de 2017, no setor de Bloco Cirúrgico, contendo uma população de 19 enfermeiros. E optou-se pela totalidade da amostra, porém considerando os critérios. $O$ de inclusão interessou-se por enfermeiros atuantes no Bloco Cirúrgico há mais de 06 meses e exclusão enfermeiro de férias, licença ou realizando carga horária complementar. A amostra final constou 15 profissionais.

Para a coleta de dados foi utilizado questionário de avaliação da Qualidade de Vida no Trabalho (QWLQ78) validado e simplificado, QWLQ-78 (REIS-JÚNIOR DR, PILATTI LA e PEDROSO B, 2011) simplificado associado a perguntas de caráter sócio demográfico. O QWLQ foi utilizado de forma simplificada, a fim de contemplar a pesquisa. Ele contém trinta e cinco perguntas e este é apresentado com cinco níveis para respostas de acordo com a Escala de Likert sendo estas: nada, muito pouco, mais ou menos, bastante $e$ extremamente. Para questionamentos do perfil sócio demográfico foram utilizadas variáveis que contemplam a opinião do profissional.

A pesquisa teve início após a aprovação do projeto, pelo Comitê de Ética e Pesquisa com CAAE 64718317.9.0000.5666 conforme a resolução $n^{\circ}$ 510/16 do Conselho Nacional de Ética em Pesquisa em Seres Humanos. Todos os pesquisados receberam um Termo de Consentimento Livre e Esclarecimento (TCLE), a fim de deixá-los cientes de tudo que lhe foi proposto.

As informações foram analisadas realizando o levantamento de dados através do Microsoft Excel ${ }^{\circledR}$ versão 2010 e Microsoft Word ${ }^{\circledR}$ versão 2010. Foi utilizada uma avaliação de estatística básica com valores absolutos e percentuais.

\section{RESULTADOS}

Ao analisar o perfil sócio demográfico da amostra desta pesquisa, percebe-se que o maior índice de profissionais enfermeiros foram $13 / 15$ (87\%) sexo feminino, o sexo masculino sendo $2 / 15$ (13\%), estes trabalhadores apresentam faixa etária predominante entre 31 a 40 anos com 8/15 (53\%), seguindo de 5/15 (33\%) com idade de 41 à 50 anos e aparece de 20 à 30 anos e 51 a 60 anos com o mesmo quantitativo de $1 / 15(7 \%)$ respectivamente.

Em relação ao quantitativo de filhos dessa equipe de enfermeiros equivaleram-se iguais nas respostas: Nenhum filho com $5 / 15$ (34\%) das respostas, filho único com 5/15 (33\%) e dois filhos com também 5/15 (33\%) dos resultados. Ainda em análise observou-se que 11/15 (73\%) destes profissionais possuem 2 vínculos empregatícios ativos, 3/15 (20\%) enfermeiros afirmam ter apenas 1 vínculo e 1/15 (7\%) retrata possuir 3 vínculos em unidades de saúde. Sobre a renda familiar da amostragem total 4/15 (27\%) admitem ter renda acima de 5 salários mínimos, outros com a mesma porcentagem de 4/15 (27\%) até 3 salários mínimos, ademais $4 / 15$ deles $(26 \%)$ tem renda de até 5 salários mínimos. A minoria $3 / 15(20 \%)$ desta equipe recebe até 4 salários mínimos. 
Além de determinar o perfil sócio demográfico da população, avaliamos a percepção sobre a qualidade de vida no trabalho (QVT) destes enfermeiros, através do questionário validado QWLQ-78, simplificado constituído por 35 questões. Verificando assim com as perguntas: "Quanto você cuida da sua alimentação?" (REIS-JÚNIOR DR, PILATTI LA e PEDROSO B, 2011) 11/15 (73\%) destes cuidam mais ou menos da sua alimentação. Houve importância sobre como cuidavam do sono, desta forma: "Quanto você cuida do seu sono?" (REIS-JÚNIOR DR, PILATTI LA e PEDROSO B, 2011) E encontramos empate em duas respostas que $5 / 15$ (33\%) relatam ter bastante cuidado, já os outros $5 / 15$ (33\%) dos participantes relatam problemas, cuidando assim muito pouco deste fator.

Tabela 1 - Análise estatística sobre as respostas das questões do questionário aplicado mostrando a comparação entre homens e mulheres.

\begin{tabular}{cccccc}
\hline Questões & Variáveis de Likert & Mulheres & Homens & Valor de $\mathbf{P}$ & $\begin{array}{c}\text { Intervalo de } \\
\text { confiança }\end{array}$ \\
\hline Q6 & mais ou menos & $09 / 13(69 \%)$ & $02 / 02(100 \%)$ & $\mathrm{P}<0,05$ & $0,4-0,8$ \\
Q30 & Bastante & $06 / 13(46 \%)$ & $01 / 02(50 \%)$ & $\mathrm{P}<0,05$ & $0,55-0,79$ \\
Q31 & Bastante & $07 / 13(54 \%)$ & $01 / 02(50 \%)$ & $\mathrm{P}<0,05$ & $1,2-2,4$ \\
Q32 & Bastante & $06 / 13(46 \%)$ & $01 / 02(50 \%)$ & $\mathrm{P}<0,05$ & $0,4-0,8$ \\
Q33 & Bastante & $08 / 13(62 \%)$ & $02 / 02(100 \%)$ & $\mathrm{P}<0,05$ & $1,2-2,4$ \\
Q35 & Bastante & $06 / 13(46 \%)$ & $01 / 02(50 \%)$ & $\mathrm{P}<0,05$ & $3,2-2,8$ \\
\hline
\end{tabular}

Fonte: Lucena MS,et al., 2017.

No caso das variáveis categóricas, empregou-se o teste estatístico de independência, qui-quadrado de Pearson. De posse dos resultados, realizamos a análise dos testes referidos anteriormente. $O$ nível de significância estabelecido para todos os testes foi de $p<0,05$.

O problema com sono em relação a prejudicar o trabalho, apontou a mais uma interrogação para estes: "Em que medida algum problema com o sono prejudica seu trabalho?" (REIS-JÚNIOR DR, PILATTI LA e PEDROSO B, 2011). Empatando em três variáveis com 12 opiniões assim dividas com: 4/15 (27\%) profissionais afirmam que o sono não prejudica em nada o seu trabalho, outros 4/15 (26\%) muito pouco e mais $4 / 15(27 \%)$ relatam afetar bastante o trabalho. Houve interesse de saber como estava à autoestima desses profissionais no trabalho com: "Com que medida você avalia sua alto-estima no trabalho?" (REISJÚNIOR DR, PILATTI LA e PEDROSO B, 2011) A resposta predominante foi mais ou menos com 7/15 (46\%) indivíduos.

A preocupação com a saúde foi um eixo que apresentaram questionamentos da seguinte forma: "Quanto você se preocupa com sua saúde?” (REIS-JÚNIOR DR, PILATTI LA e PEDROSO B, 2011) 6/15 (40\%) dos trabalhadores demonstrarem a resposta mais ou menos para cuidar-se. "Em que medida você tem dificuldade para cuidar da sua saúde?" (REIS-JÚNIOR DR, PILATTI LA e PEDROSO B, 2011) 6/15 (40\%) dos pesquisados referirem muito pouco o nível de dificuldade para não se cuidar.

Em relação a ficar doente devido ao local de trabalho, foi perguntado: "Com que frequência você fica doente devido ao seu trabalho?" (REIS-JÚNIOR DR, PILATTI LA e PEDROSO B, 2011) 9/15 (60\%) relatam que muito pouco, ficam doentes devido a suas atividades. $\mathrm{E}$ ainda perguntamos: "Você pratica exercícios físicos regulares?' (REIS-JÚNIOR DR, PILATTI LA e PEDROSO B, 2011) 8/15 (53\%) dos entrevistados não praticam nada de atividade física regular na sua rotina diária. Sobre a qualidade do lazer que eles dispõem, com a abordagem a seguir: "Em que medida você avalia a qualidade do lazer e da sua família?" (REISJÚNIOR DR, PILATTI LA e PEDROSO B, 2011) 6/15 (40\%) classificam como mais ou menos, tendo esta como resposta predominante.

A preocupação com dores e desconfortos no trabalho da enfermagem, foi um fator considerável para ser analisado: "Quanto você se preocupa com dores ou desconfortos no trabalho?" (REIS-JÚNIOR DR, PILATTI LA e PEDROSO B, 2011). Teve classificação como mais ou menos por $5 / 15$ (33\%) integrantes da equipe. 
Sobre a carga horária de trabalho diário interrogamos: "Como você avalia a sua carga horária de trabalho diário?" (REIS-JÚNIOR DR, PILATTI LA e PEDROSO B, 2011) Duas respostas equivalentes com 5/15 (33\%) afirmações de mais ou menos e outros $5 / 15$ (33\%) profissionais definiram ser bastante a carga horária de trabalho diário. Perguntamos se algum sentimento negativo infere no trabalho destes enfermeiros da seguinte forma: "Em que medida algum sentimento negativo (tristeza, desespero) interfere no seu trabalho?" (REISJÚNIOR DR, PILATTI LA e PEDROSO B, 2011) E 7/15 (47\%) disseram como resposta muito pouco na proporção de likert.

A cooperação entre os níveis hierárquicos relatados pela seguinte pergunta: "Como você avalia a cooperação entre os níveis hierárquicos no trabalho?" (REIS-JÚNIOR DR, PILATTI LA e PEDROSO B, 2011) Obteve empate entre duas variáveis com 6/15 (40\%) alegações para muito pouco e outras 6/15 (40\%) para bastante a analise deles. Do mesmo modo a igualdade de tratamento entre os funcionários esteve como um fator importante a se fazer questionamento: "Como você avalia a igualdade de tratamento entre funcionários?" (REIS-JÚNIOR DR, PILATTI LA e PEDROSO B, 2011) Apresentou a mesma quantidade na resposta com $5 / 15(33 \%)$ funcionários mencionando mais ou menos a igualdade e $5 / 15(34 \%)$ dos demais trazem que há bastante igualação no tratamento.

A motivação para trabalhar desta equipe de enfermagem, conteve também no questionário: "Em que medida você avalia sua motivação para trabalhar?" (REIS-JÚNIOR DR, PILATTI LA e PEDROSO B, 2011) A predominância existiu em ser bastante motivados com 5/15 (33\%) em sua definição. Estes enfermeiros do bloco cirúrgico foram interrogados sobre a satisfação com o nível de participação nas decisões da empresa, deste modo: "Em que medida você está satisfeito com o seu nível de participação nas decisões da empresa?" (REIS-JÚNIOR DR, PILATTI LA e PEDROSO B, 2011) 4/15 (27\%) declararam que estão nada satisfeito, e $4 / 15(27 \%)$ opostos citaram estar com bastante satisfação.

A obrigação de mudança de rotina em casa, devido ao trabalho foi questionada se era frequente na vida desta equipe da área de saúde da seguinte maneira: "Com que frequência você é obrigado a mudar sua rotina em casa devido ao trabalho?" (REIS-JÚNIOR DR, PILATTI LA e PEDROSO B, 2011) Como resultado, três das 5 opções de variáveis tiveram mesmo índice de opinião, com 4/15 (27\%) dos trabalhadores da equipe de enfermagem apoiando nada, $4 / 15$ (27\%) diferentes ficaram com a opção mais ou menos e os outros $4 / 15$ $(26 \%)$ afirmando que são bastantes obrigados a mudar a rotina em casa devido suas atividades laborais.

Porém apesar disso na analise da questão: "Você se sente realizado com o que faz?" (REIS-JÚNIOR DR, PILATTI LA e PEDROSO B, 2011) 7/15 (46\%) consideram-se bastante realizados com o que fazem. Contudo quando questionados em relação a mudar de emprego: "Com que frequência você pensa em mudar de emprego radicalmente?' (REIS-JÚNIOR DR, PILATTI LA e PEDROSO B, 2011) 6/15 (40\%) não pensa nada frequente em mudar radicalmente de emprego, mas $6 / 15$ (40\%) dos demais representantes da classe pensam muito pouco nesta possibilidade. Todavia na verificação da pergunta: "Em que medida você avalia o orgulho pela sua profissão?" (REIS-JÚNIOR DR, PILATTI LA e PEDROSO B, 2011) 9/15 (60\%) da equipe de enfermeiros medem como bastante o orgulho pela profissão. A qualidade de vida no trabalho também foi avaliada: "O quanto você está satisfeito com sua qualidade de vida no trabalho?" (REIS-JÚNIOR DR, PILATTI LA e PEDROSO B, 2011) 9/15 (60\%) funcionários classificaram de mais ou menos.

Relacionado à segurança quanto à prevenção de acidentes de trabalho: "Em que medida você se sente seguro quanto à prevenção de acidentes de trabalho?" (REIS-JÚNIOR DR, PILATTI LA e PEDROSO B, 2011) O resultado obteve igualdade em 3 alternativas, 4/15 (27\%) enfermeiros alegaram nada seguro, 4/15 (27\%) divergentes optaram por muito pouco e $4 / 15$ (26\%) deles relatam ser bastante seguros quanto a prevenção de acidentes.

A estabilidade no emprego foi outro fator abordado: "Você se sente estável no seu emprego?" (REISJÚNIOR DR, PILATTI LA e PEDROSO B, 2011) 6/15 (40\%) líderes da enfermagem afirmaram sentir bastante estável em seu emprego neste hospital onde exercem suas atividades. Quando foi citada a avaliação destes perante a remuneração pelo trabalho: "Como você avalia a sua remuneração pelo trabalho?" (REIS-JÚNIOR DR, PILATTI LA e PEDROSO B, 2011) 13/15 (87\%) enfermeiros distribuíram a preferência à resposta muito pouco. Consideramos também a importância de conhecer a frequência que estes indivíduos necessitam de 
outras fontes de dinheiro para se sustentar no questionamento: "Com que frequência você necessita de outras fontes de dinheiro pra se sustentar?” (REIS-JÚNIOR DR, PILATTI LA e PEDROSO B, 2011) 7/15 (46\%) dos entrevistados referem bastante à necessidade de outras rendas.

O apoio dos colegas é um fator que contribuí para o trabalho, assim o questionário realiza esta pergunta: "Você consegue dos colegas o apoio que necessita no trabalho?" (REIS-JÚNIOR DR, PILATTI LA e PEDROSO B, 2011) 8/15 (53\%) gerentes da classe informaram que conseguem bastante o apoio necessário. Outra causa que gera reflexão nos profissionais enfermeiros: "Ao final da jornada de trabalho, o quanto você se sente cansado?" (REIS-JÚNIOR DR, PILATTI LA e PEDROSO B, 2011) 7/15 (47\%) relataram que ao final da jornada de trabalho sentem-se bastante cansados.

Os enfermeiros responderam no questionário a questão: "Em que medida você gosta do nível de desafio que the é proposto no trabalho?" (REIS-JÚNIOR DR, PILATTI LA e PEDROSO B, 2011) 10/15 (67\%) avaliaram gostar bastante do nível de desafio que the é proposto no trabalho. Correlacionando a outra pergunta constituída neste questionário QWLQ- 78 Simplificado a cerca de: "Você se sente satisfeito com a variedade de tarefas que realiza?" (REIS-JÚNIOR DR, PILATTI LA e PEDROSO B, 2011) 7/15 (47\%) destes tratam como bastante satisfeitos com suas variedades.

$\mathrm{Na}$ interrogação a respeito: "Em que medida você sente orgulho da organização onde trabalha?" (REISJÚNIOR DR, PILATTI LA e PEDROSO B, 2011) Obtivemos três alternativas com resultados iguais, assim $4 / 15(27 \%)$ profissionais escolheram a alternativa muito pouco, $4 / 15$ (27\%) a de mais ou menos e $4 / 15(26 \%)$ discordando dos demais e relatando possuir bastante orgulho da unidade hospitalar na qual trabalha.

\section{DISCUSSÃO}

A pesquisa tratou-se analisar percepção de QVT em enfermeiros atuantes no bloco cirúrgico. Referente à determinação sócio demográfica, constatou-se predominância feminina, de faixa etária média, a maioria possuindo dois vínculos empregatícios, com variáveis iguais nos resultados de filhos e renda familiar. Assim, houve equivalência em três alternativas (AKTER $N$ et al., 2017). Em relação à remuneração, jornada de trabalho e quantidade de vinculação, evidencia sobrecarga de trabalho por referirem baixa remuneração, contudo necessidade de possuir mais de um emprego (GUIMARÃES ALO e FELLI VEA, 2016).

A qualidade de vida no trabalho se diz respeito a tornar o espaço onde exerce as suas atividades ainda mais humano, ocasionando o levantamento de prós e contra entre trabalho e vida pessoal. Com a realização de pesquisas e respectivamente seu resultado Walton em 1973 definiu oito subdivisões: Compensação justa e adequada; Condições de trabalho avaliando a jornada de trabalho exercida pelo profissional; Desenvolvimentos das capacidades de acordo com autonomia e autodomínio; Chances de crescimento pessoal e segurança dentro da organização; Integração social na empresa possibilitando oportunidades equivalentes; Constitucionalismo acatando as leis trabalhistas; Trabalho e espaço total de vida com solidez nos horários; Relevância social no trabalho (LOPES ECS, 2015).

A listagem de perguntas evidenciou a percepção dos enfermeiros sobre sua Qualidade de Vida no Trabalho (QVT), e apresentou um valor considerável de 60\% estarem mais ou menos satisfeitos com esta. Trabalhar no indivíduo esta temática é uma importante estratégia, pois pode induzir positivamente gerando satisfação, motivação e comprometimento, contudo, negativamente, se não acompanhado e instruído, provoca insatisfação no ambiente de trabalho. O emprego além de ser extraído o faturamento, permite obter satisfação por efetuar algo produtivo, além de proporcionar sensação de pertencer a uma equipe (KOWITLAWKUL Y et al., 2018; SHOJI Set al., 2016).

Assim, o fator salário proposto à classe apresentou a pior avaliação nos índices, atingindo $87 \%$ para insatisfação. Por este motivo entendemos que os profissionais enfermeiros apontaram que são mal remunerados. Com esta realidade, diversos trabalhadores são submetidos à opção de possuir mais de um vinculo, abdicando o seu tempo destinado a descanso (SARTORETO IS e KURCGANT P, 2017). Desta maneira, os enfermeiros podem deixar de sentir prazer no trabalho, por desencadearem cansaço físico e mental, acarretando angústia com a profissão (GUIRAMARÃES ALO e FELLI VEA, 2016). 
Os enfermeiros alegarem que é importante o trabalho em equipe e 53\% dos trabalhadores afirmaram na entrevista obterem bastante essa cooperação no campo de atuação com os diferentes profissionais. Quando usamos o termo de trabalho em equipe, faz referencia a trabalhar com interação, construindo consenso no que vai ser executado, pois esse tipo de atividade requer cooperação, compromisso e responsabilidade gerando harmonia e respeito mútuo (SANTOS JLG et al., 2016).

De acordo com os participantes da pesquisa, $46 \%$ deles referiram realização no trabalho que executam. A definição de satisfação profissional está relativamente caracterizada à maneira como o profissional se sente no momento em que executa suas atividades.

A satisfação é a razão de sucesso profissional, e quando insatisfeito pode desencadear doenças que possam atingir a saúde deste trabalhador, conhecida como doenças ocupacionais ou apresentar manifestações de síndromes relacionadas ao estresse (SOUZA AC MILANI D e ALEXANDRE NMC, 2015).

Não surpreende $47 \%$ estarem bastante cansados ao final da jornada de trabalho. Pois, maior parte do tempo dos enfermeiros é empregado em instituições hospitalares. A QVT destes pode ser reduzida por não atender necessidades de trabalho por quantidade ou qualidade de fatores e se relacionar a circunstancia encontrada como o sedentarismo, consegue interferir no processo saúde doença (CRUZ JP et al., 2018).

Quanto ao período de tempo destinado ao repouso e lazer, estes avaliam como mais ou menos este tempo. Nestes momentos como lazer e família, importante evitar que atividades profissionais ou domésticas ocupem maioria desse tempo (KELBISO L, BELAY A e WOLDIE M,2017).

$\mathrm{Na}$ analise de mau hábito alimentar e sedentarismo, responderam $53 \%$ para nada de pratica de atividade física e $73 \%$ disse como resposta mais ou menos para cuidado com alimentação. Assim, argumentaram precariedade de tempo e alguns informaram a falta de estimulo.

Entretanto, referindo-se a trabalhadores de enfermagem, e estes entenderem os benefícios de uma boa alimentação e a importância da prática regular de atividade física, e não aderirem a estes hábitos que podem causar o desencadeamento de Doenças Crônicas Não Transmissíveis (DCNT), como obesidade, hipertensão arterial, doenças cardiovasculares e diabetes mellitus e ainda interferir na sua qualidade de vida no trabalho (FREIRE CB et al., 2015).

Requisito importante é o orgulho por onde trabalha. Nos resultados, três respostas empataram: muito pouco, mais ou menos e bastante. Também houve questionamento sobre fatores motivacionais, obtendo-se resultado de $33 \%$ em sentirem bastante motivados. Estes itens possuem correlação satisfação/motivação. Promover local de trabalho ligado a estas 2 categorias é relevante, pois o profissional desempenhará com qualidade seu exercício, reduzindo danos aos profissionais e à organização. Há também garantia da QVT na vida pessoal e familiar (PEREIRA GC e ROMÃO LN, 2018, ABBASI M, 2017).

Esta pesquisa contribuiu para melhorar a percepção da QVT do enfermeiro e demonstra a importância em investir em melhorias do trabalho que busquem prevenção da saúde em progresso da QVT. Ressalta-se que este estudo possui limitação por ser feito apenas com profissional de nível superior da enfermagem atuante em bloco cirúrgico, pois na graduação haveria pouco tempo para coleta, dado o número elevado de questões do instrumento de coleta e profissionais de nível técnico, assim, não foi observado a visão destes trabalhadores da equipe de enfermagem.

\section{CONCLUSÃO}

Os resultados demonstram percepção de trabalhadores de enfermagem sobre sua QVT, à maioria reconhece como mais ou menos segundo a escala de likert, mostrando assim comprometida, apesar de revelarem sentimento de satisfação pela profissão e tarefas que executam. Porém, desarmoniza este entusiasmo quando expõem circunstâncias de insatisfação salarial. Diante disso, é essencial que estes reconheçam o que possa ser alterado no estilo de vida atual para melhoria de sua QVT. Portanto, merece destaque a organização provê melhores condições relacionadas à quantidade e qualidade na estrutura como um todo, além de melhoria salarial para sentirem-se realizados e valorizados no ambiente de trabalho. Entretanto, tomadas estas medidas estes desempenharão uma auto avaliação melhor de qualidade de vida. 


\section{REFERÊNCIAS}

1. ABBASI M. Investigation of the Relationship between Work Ability and Work-related Quality of Line in Nurses. Iranian Journal of Public Health. 2017; 46(10) 1404-1412.

2. ADRIANO LGO. Métodos que definem a Qualidade de Vida no Trabalho do enfermeiro na Urgência e Emergência. Revista UNINGÁ Review. 2017; 20(2) 30-36.

3. AKTER N, et al. Factors predicting quality of work life among nurses in tertiary-level hospitals, Bangladesh. International Nursing Review. 2017.

4. CRUZ JP, et al Optimism, Proactive Coping and Quality of Life among Nurses: A Cross-Sectional Study. Journal of Clinical Nursing. 2018.

5. FREIRE CB, et al. Qualidade de vida e atividade física em profissionais de terapia intensiva sub médio São Francisco. Rev. Bras. Enferm. 2015; 68 (1) 1-8.

6. FU CY, et al. Associations of professional quality of life and social support with health in clinical nurses. J Nurs Manag. 2018; 26(2) 172-179.

7. GUIMARÃES ALO, FELLI VEA. Notificação de problemas de saúde em trabalhadores de enfermagem de hospitais universitário. Rev. Bras. Enferm. 2016; 69 (3) 507-514.

8. KELBISO L, et al. Determinants of Quality of Work Life among Nurses Working in Hawassa Town Public Health Facilities, South Ethiopia: A Cross-Sectional Study. Nursing Research and Practice. 2017.

9. KOWITLAWKUL Y, et al. Ivestigating nurses' quality of life and work-life balance statuses in Singapore. International Nursing Review. 2018.

10. LOPES ECS. Qualidade de vida no trabalho segundo os servidores do Centro de Pesquisa René RachouCPqRR/Fiocruz [Dissertação de mestrado em saúde pública]. Rio de Janeiro- RJ: Escola Nacional de Saúde Pública Sergio Arouca/Fiocruz; 2015.

11. PEREIRA GC, ROMÃO LN. Quality of Life at Work: A study conducted in an organization of waste collection not dangerous. Research, Society and Development. 2018; 7(5) 1-15.

12. REIS-JÚNIOR DR, et al. Qualidade de vida no trabalho: construção e validação do questionário QWLQ78. Revista Brasileira de Qualidade de Vida. 2011; 03(02) 1-11.

13. REZENDE KJ, GUARDA RM. Qualidade de vida no trabalho dos garis de Palmas-TO. Revista Multidebates. 2017; $1(2)$.

14. SANTOS JLG, et al. Estratégias utilizadas pelos enfermeiros para promover trabalho em equipe em um serviço de emergência. Rev. Gaúcha de Enferm. 2016; 37 (1) 1-8.

15. SARTORETO IS, KURCGANT P. Satisfação e Insatisfação no trabalho do Enfermeiro. Revista Brasileira de Ciências da Saúde. 2017; 21(2) 181-188.

16. SHOJI S, et al. Proposta de melhorias de trabalho em uma unidade ambulatorial: perspectiva da enfermagem. Esc. Anna Nery 2016; 20(2) 1-9.

17. SOUZA AC, et al. Adaptdão cultural de um instrumento para avaliar a satisfação no trabalho. Rev. bras. saúde ocup. 2015; 40 (132) 1-10. 